\title{
Directed Cooperation in Multihop Wireless Sensors Network
}

\author{
Jan Nikodem, Ryszard Klempous, Maciej Nikodem, Marek Woda and Zenon Chaczko
}

\begin{abstract}
This paper proposes a relational abstraction for Wireless Sensors Network where node can identify its neighbors around it. Because of limited radio link range only some of nodes have a direct contact with the base station and transmission is carried out in a multihop way so information is send from one node to another towards the BS. We propose a relation $\pi$ that represents cooperation between nodes and takes advantages of topological properties of the network. Based on the hop-distance from the BS any node $k$ can determine a set $N_{<}(k)$ that consists of nodes to which $k$ should send messages in order to retain a data-flow direction towards the BS.
\end{abstract}

Keywords-Wireless sensors network, directed cooperation, relations.

\section{INTRODUCTION}

W IRELESS Sensors Network (WSN) is a wireless network spatially distributed over some area that consists of autonomous devices (sensors, nodes) monitoring and transmitting physical and environmental conditions. Each device is typically equipped with microcontroller, one or more sensors (e.g. temperature, humidity, accelerometer, etc.), radio transceiver, and energy source. Typically, energy required for sensor to operate is derived from batteries which cause the energy to be one of the most important constrains. Because of the spatial distribution and constrained resources, operation of sensors is focused on local activities and mutual communication. This enables the WSN to perform tasks that single sensor cannot afford causing communication to be one of the most crucial activities for a node. Information gathered by sensors in area is transmitted over to the base station (BS). Due to power constrains, and relatively short communication ranges of each node, transmission is carried out in a multihop way so information is send from one node to another towards the BS. Transmitting information towards the BS seems to be achievable easily but in fact it is a challenging task for nodes distributed over large area exceeding communication range of a single sensor. Uncontrolled information broadcasting is not a solution too, since it consumes communication bandwidth, leads to collisions in communication channel and increases overall power consumption. Due to fulfill constrains it is

This work was supported by E.U. Regional Development Fund and by Polish Government within the framework of the Operational Programme - Innovative Economy 2007-2013. Contract POIG.01.03.01-02-002/08-00, Detectors and sensors for measuring factors hazardous to environment modeling and monitoring of threats.

J.Nikodem, R.Klempous, M.Nikodem and M.Woda, Faculty of Electronics, Wrocław University of Technology, Wyspianskiego 27, 50-370 Wrocław, Poland (e-mail: \{jan.nikodem, ryszard.klempous, maciej.nikodem, marek.woda\}@pwr.wroc.pl).

Z.Chaczko, University of Technology Sydney, PO Box 123, Broadway NSW 2007, Australia (e-mail: zenon.chaczko@uts.edu.au). required that information flows from nodes to the BS in a way that minimizes power consumption, rather than being transmitted to all nodes in the network. Additionally, when the communication is controlled in a static manner (e.g. the shortest transmission path is chosen) then the network performance, its dependability and life time can be reduced due to unequal bias of some sensors.

Our paper focuses on so called spatial communication that outdo path based communication and aims to construct multiple communication paths towards the BS, that information can be send through. Construction of these paths is based on local information gathered by a node that received an information. Based on this knowledge the node decides where to send the information. This allows sharing communication costs among multiple communication paths and nodes. Moreover, it increases communication robustness and safety since communication path can be changed in case of an interferences or node captures.

\section{PRoblem Description}

Multihop routing path construction requires cooperation of neighborhood nodes in order to establish a communication path. Regardless of whether one or multiple paths are constructed, routing algorithms aim to construct a BS-oriented communication path. Therefore, operation of a single node during routing path discovery is focused on reaching the BS. Additional criteria (like minimal energy costs, minimal number of hops, etc.), expressed in terms of a function to be minimized, help the node to chose a right way to route information. This is quite the opposite comparing to spatial communication that takes advantages of relations instead of functions. There is no precise decision on subsequent nodes to which the information should be send. Instead, fulfillment of certain relation between nodes is required when establishing spatial communication paths. This assumes that nodes cooperate locally within the neighborhood using local criteria and taking local decisions. This clearly leads to concerns about how to determine criteria for local optimization in such a way, that the resulting spatial communication path satisfies criteria defined globally.

Relation-based cooperation between nodes takes advantages of topological properties of the network and is not oriented towards any particular direction - nodes simply cooperate within a neighborhood. However, the whole WSN is focused on collecting information from sensors and sending it down to the BS. Therefore, nodes that operate within a neighborhood have to ensure that based on locally taken decisions they can 
establish multihop routing paths from network nodes towards the BS. In this paper we present how to implement relationoriented cooperation within a neighborhood and based on locally taken decisions construct spatial communication path that fulfills globally defined criteria.

\section{RELATED WORK}

There are several routing algorithms for WSNs that allow sending information between nodes and the BS. So called flat algorithms (i.e. flooding) ensure that every information will finally reach the BS unless there is no node that can communicate to the BS directly [1], [3]. Such protocols use broadcast communication and simply flood the whole network with any information causing collisions in the communication channel and retransmissions. Apart from the waste of energy, flooding also decreases the bandwidth, lowers communication speed or simply cuts of some parts of the network. These drawbacks are minimized in gossip protocol [5] where the broadcast communication concerns only neighboring nodes. Such routing protocols as Data Centric Routing (DCT), Sensor Protocol for Information via Negotiation (SPIN) try to orient communication towards time, query, event (DCT) or information requirements (SPIN). Directed Diffusion protocol enables to minimize energy consumption during the routing protocol further but a lower efficiency (mainly due to large number of queries) is its drawback. Rumor Routing [1] is another attempt to take advantage of agents and directed communication. This gives good results for a limited number of situations when costs of additional agents are negligible comparing to the overall costs. Hierarchical protocols, such as LEACH, TEEN and PEGASIS [9], [13] aim at directed communication that is achieved through clustering or divisions into zones. This proposal use routing protocols based on tree or chain structures.

All aforementioned protocols construct a single multi-hop communication path between any node and the BS. Established communication path is utilized as long as it is energetically justified [6], [8]. If energy level of a node that belongs to the path, drops below specified level then communication path discovery algorithm is being run again. The whole process is then repeated until no path can be found.

\section{RELATIONS, CHAINS OF ACTIONS}

Our approach utilizes three basic binary relations defined on the set of actions (Act) that describes communication activities in WSN. These three relations: collision $(\varkappa)$, subordination $(\pi)$ and tolerance $(\vartheta)$, were first introduced and described by Jaron [7] and later by Nikodem et al. [11], [10]. Any binary relation $\mathcal{R}$ on set $A$ can be represented as a set of ordered pairs $\langle x, y\rangle$ where $x, y \in A$ and $x, y$ are in relation $\mathcal{R}$ (which we denote $x \mathcal{R} y$ ):

$$
\mathcal{R}=\{\langle x, y\rangle \mid x, y \in A, x \mathcal{R} y\} .
$$

Thus one may treat relation as a set such that $\mathcal{R} \subset A \times A$. We may also define the converse of relation $\mathcal{R}$ as:

$$
\left.\mathcal{R}^{-1}=\{<x, y\rangle \mid x, y \in A, y \mathcal{R} x\right\} .
$$

Based on these definitions we can describe basic properties of $\varkappa, \pi$ and $\vartheta$ as follows [7]:

$$
\pi \cup \vartheta \cup \varkappa \subset \text { Act } \times \text { Act } \neq \emptyset,
$$

and

$$
\iota \cup(\pi \circ \pi) \subset \pi,
$$

where $\iota$ is a identity relation on the set Act. Formula (3) states that all three relations are binary on non-empty set of Actions. Formula (4) states that subordination is reflexive $(\iota \subset \pi)$ and transitive $(\pi \circ \pi \subset \pi)$. Further

$$
\pi \cup \vartheta^{-1} \cup(\vartheta \circ \pi) \subset \vartheta
$$

means that:

- subordination implies tolerance - if $\pi$ holds for some $x, y \in$ Act then $\vartheta$ also holds for these,

- tolerance is symmetric - if $x \vartheta y \Rightarrow y \vartheta x$,

- subordinated action tolerates all actions tolerated by the dominant - if $(x \pi y \wedge y \vartheta z) \Rightarrow x \vartheta z$.

For collision relation we have that

$$
\varkappa^{-1} \cup\{\pi \circ \varkappa\} \subset \varkappa \subset \vartheta^{\prime}
$$

where $\vartheta^{\prime}$ is the complement of $\vartheta$ :

$$
\left.\vartheta^{\prime}=\{<x, y\rangle \in X \times Y|<x, y\rangle \notin \vartheta\right\} .
$$

Axiom (6) states that collision is symmetric, disjoint to tolerance and subordinated action must be in collision with action being in collision with dominant. Paper [10] presents how to use relations $\pi, \vartheta$ and $\varkappa$ in order to model spatial communication. Subordination $\pi$ is responsible for multihop path determination, tolerance $\vartheta$ allows multiple paths to exist simultaneously, while collision $\varkappa$ forms restrictions for the communication space. This paper focuses on subordination

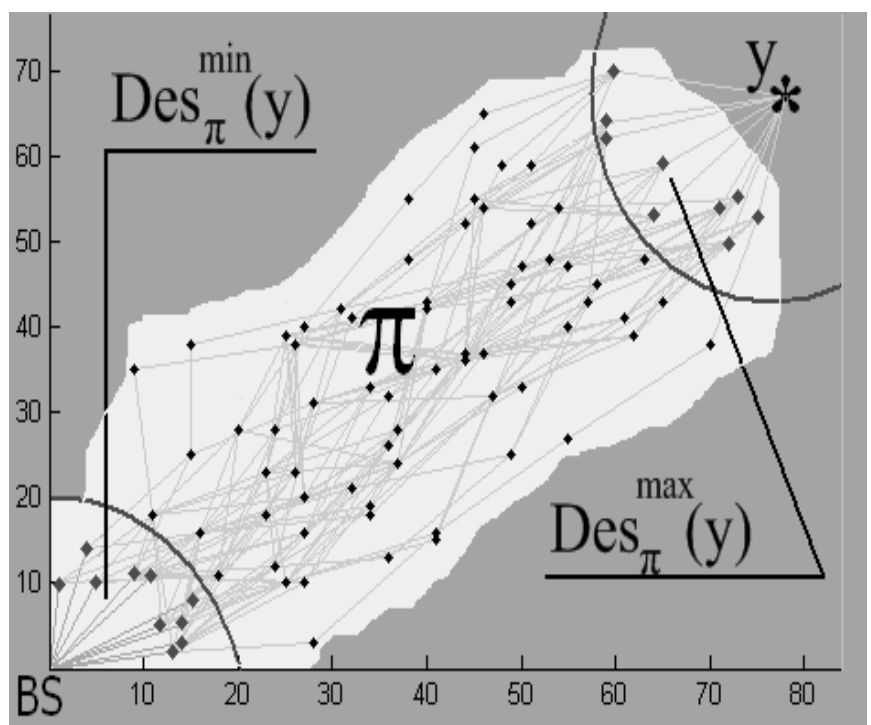

Fig. 1. $\operatorname{Des}_{\pi}(\mathbf{y})$ area in WSN.

since among other two relations it is the most important for determination of a multihop communication path. It is also the only one relation that is transitive enabling to construct chains of WSN's nodes that will establish communication paths. 
Because $\pi$ is transitive we can define chain of subordination relation $\pi^{n}$ :

$$
\pi^{n}=\pi^{n-1} \circ \pi ; \quad \ldots \quad \pi^{2}=\pi^{1} \circ \pi ; \quad \pi^{1}=\pi .
$$

Then

$$
\pi^{n}=\left\{<x, y>\mid<x, y>\in A c t^{n-1} \times A c t\right\}
$$

where $x$ is the nearest descender of $y$ (or $y$ is the nearest ascender of $x$ ).

Using composition of relation is more troublesome comparing to set of node $y$ descenders:

$$
\operatorname{Des}_{\pi}(y)=\left\{x \in A c t \mid(\exists n \in N)\left(x \pi^{n} y\right)\right\}
$$

We can take many subsets of $\operatorname{Des}_{\pi}(y)$ but only some of them will be useful in future investigations. Three of them are the most important ones:

$$
\begin{gathered}
\operatorname{Des}_{\pi}^{\text {min }}(y)=\left\{x \in \operatorname{Des}_{\pi}(y) \mid(B S \pi x)\right\} \\
\operatorname{Des}_{\pi}^{m i s}(y)=\left\{x \in \operatorname{Des}_{\pi}(y) \mid \neg\left(\exists z \in D e s_{\pi}\right)(z \pi x)\right\} \\
\operatorname{Des}_{\pi}^{\text {max }}(y)=\left\{x \in D e s_{\pi}(y) \mid(x \pi y)\right\}
\end{gathered}
$$

The (11) is a set of the nearest ascenders of the base station while the (13) is a set of nearest descenders of $y$ (fig.1). From remaining subsets of $\operatorname{Des}_{\pi}(y)$ we will focus only on these that refer to multi-hop communication path between node $y$ and the BS. We will denote these subsets as $C h n_{\pi}(y)$ and we will construct the subordination relation in order to maximize the cardinality of $D e s_{\pi}^{\min }(y)$ and $C h n_{\pi}(y)$.

To construct a proper subordination relation, well suited for WSN communication path modeling, we have to remember that according to eq. (4) relation $\pi$ is transitive $((\pi \cdot \pi) \subset \pi)$ and reflexive $(\iota \subset \pi)$. In case two nodes of the network are situated close to each other we usually assume that they "glue" together forming a single node of communication:

$$
(\forall x, y \in A c t)(x \pi y \wedge y \pi x\} \Rightarrow x=y),
$$

which means that subordination becomes antisymmetric. Moreover, in WSN it is obvious that node does not send message to itself. Hence for modeling communication activity we refine subordination as irreflexive.

$$
(\forall x \in A c t)(\neg(x \pi x)) .
$$

Note that combination of transitivity and irreflexivity implies that if $x \pi y$ holds, then $\neg y \pi x-y$ is not subordinated to $x$. In this way we obtain a stronger asymmetric property comparing to $(14)$

$$
(\forall x, y \in A c t)(x \pi y \Rightarrow \neg(y \pi x)) .
$$

This property ensures that there are no loops in $C h n_{\pi}(y)$ chains and relation $\pi$ is directed. According to the aforementioned requirements (transitivity, (16), (15)) for the relation $\pi$ we get that subordination is a strict ordering relation on a set of actions $\operatorname{Des}_{\pi}(y)$. Nevertheless, $\pi$ does not partition the set $\operatorname{Des}_{\pi}(y)$ but as a result we obtain linear ordered chains $C h n_{\pi}(y)$.

Due to limited communication range of a single node smaller then the size of the WSN, the relation $\pi$ is never total. Generally, concerning relation $\pi$, intensity quotient within multi-hop network is rather low. However, if we consider this quotient within node's neighborhood then the range of possible values is expanded.

\section{CoOperation Within NeIghborhoods}

Cooperation is crucial activity in a multi-hop WSN [4], [14]. Most nodes do not communicate directly with the base station because it is out of node's radio communication range. WSN is really distributed, nodes provide necessary computations, communicate with each other within some neighborhood, and maintain state of the whole network.

There are many WSN algorithms which take advantage of some concept of a neighborhood (e.g. multi-hop, reliable, bi-directional or geographic). That is why we start from neighborhood definition. Let us define $\operatorname{Map}(X, Y)$ as a set of mapping functions from $X$ onto $Y$ (surjection). Where $\operatorname{Sub}(X)$ is defined as a family of all $X$ subsets. We define the neighborhood $\mathcal{N}$ as follows

$$
\mathcal{N} \in \operatorname{Map}(\text { Nodes, Sub(Nodes })) .
$$

Thus, $\mathcal{N}(k)$ is the neighborhood of node $k$, and $\mathcal{N}(S)$ is the neighborhood of $S$ (set of nodes) defined as:

$$
\begin{gathered}
\mathcal{N}(k)_{\left.\right|_{k} \in \text { Nodes }}=\left\{y \in \text { Nodes } \mid y \mathcal{R}_{\mathcal{N}} k\right\}, \\
\mathcal{N}(S)_{\left.\right|_{S} \subset \text { Nodes }}=\left\{y \in \text { Nodes } \mid(\exists x \in S)\left(y \mathcal{R}_{\mathcal{N}} k\right)\right\} .
\end{gathered}
$$

Paper [12] analyses different definitions of locality that can be found in the literature. It also presents advantages and drawbacks of dividing network into clusters (clustering), single routing path and neighborhoods. Native locality has been pinpointed as the one, to work with neighborhood abstraction that is determined by native (mostly technical) constraints like radio link range. Whereas clustering is some kind of simplification, that facilitates computation and truncates the set of possible solutions at the same time. As the result, the native neighborhood was advised as the most suitable form of the local range. Considering native neighborhoods within WSN network they are indexed family of sets $\mathcal{N}=\left\{N_{i} \mid i \in I\right\}$ for which following properties hold:

$$
\begin{gathered}
(\forall i \in I)\left(N_{i} \neq \emptyset\right) \wedge \bigcup N_{i}=\text { Nodes } \\
(\forall i, j \in I \mid i \neq j)\left(N_{i} \bigcap N_{j} \neq \emptyset\right),
\end{gathered}
$$

which relate to local (at any node) condition:

$$
(\forall y \in N o d e s)\left(\exists^{\sim} i \in I \mid y \in \bigcap N_{i} \neq \emptyset\right),
$$

formulated here a little stronger than (21) because $\exists \sim$ means exist as many as feasible". It means that native neighborhoods do not divide the set of WSN nodes into mutually exclusive subsets.

Having well defined one-hop (native) neighborhoods, let us decompose globally defined chains in $\operatorname{Des}_{\pi}(y)$ for identical local task ascribed to each node. Once WSN is being created, during self-organizing process, distance between nodes and BS is being calculated - expressed by a number of necessary hops to reach the BS. The procedure begins with a broadcast message 'my status: 0-hops-to-BS' sent from BS. Every node 


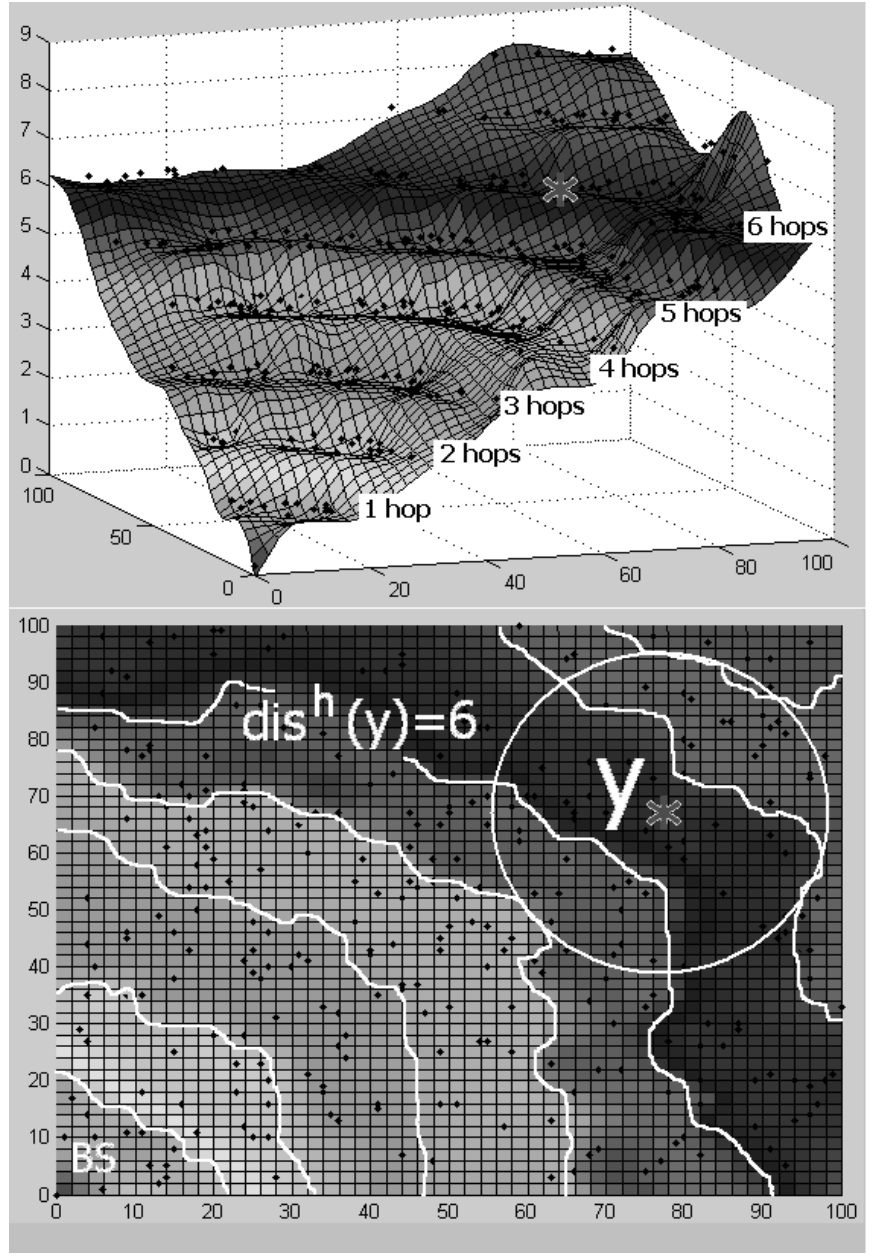

Fig. 2. Distance from $B S(0,0)$ (measured in hops) and $\operatorname{dis}^{\mathbf{h}}()$ zones.

that will hear the 'my status: X-hops-to-BS' message for the first time sets its status to ' $(\mathrm{X}+1)$-hops-to-BS' and broadcasts the 'my status: $(\mathrm{X}+1)$-hops-to-BS' message. When this procedure is finished every node with status $\mathrm{X}+1$ can communicate with at least one node with status $\mathrm{X}$. As a result any node $k$ knows its hop distance from the BS $\left(\operatorname{dis}^{h}(k)\right)$ and distances of all of its neighbors $\left(d i s^{h}(i)\right.$ where $i \in N(k)$, fig.2). Based on this information it is possible to split neighborhood $N(k)$ into three sets:

$$
\begin{aligned}
& N_{<}(k)=\left\{y \in N(k) \mid \operatorname{dis}^{h}(y)<\operatorname{dis}^{h}(k)\right\} \\
& N_{=}(k)=\left\{y \in N(k), y \neq k \mid \operatorname{dis}^{h}(y)=\operatorname{dis}^{h}(k)\right\} \\
& N_{>}(k)=\left\{y \in N(k) \mid \operatorname{dis}^{h}(y)>\operatorname{dis}^{h}(k)\right\}
\end{aligned}
$$

which are mutually exclusive and collectively exhaustive $N(k)$ but they are not partition of a set $N(k)$, because of

$$
(\exists k \in W S N)\left(N_{<}(k)=\emptyset \vee N_{>}(k)=\emptyset \vee N_{=}(k)=\emptyset\right) .
$$

Considering relation $\pi$, node's $k \in \operatorname{Des}_{\pi}(y)$ communication activity within neighborhood becomes limited to:

$$
N_{\pi}(k)=\{y \in N(k) \mid y \pi k\}
$$

Knowledge of the set $N_{<}(k)$ (23) allows a node $k$ to determine elements of the sets (25) in order to retain a data-flow direction

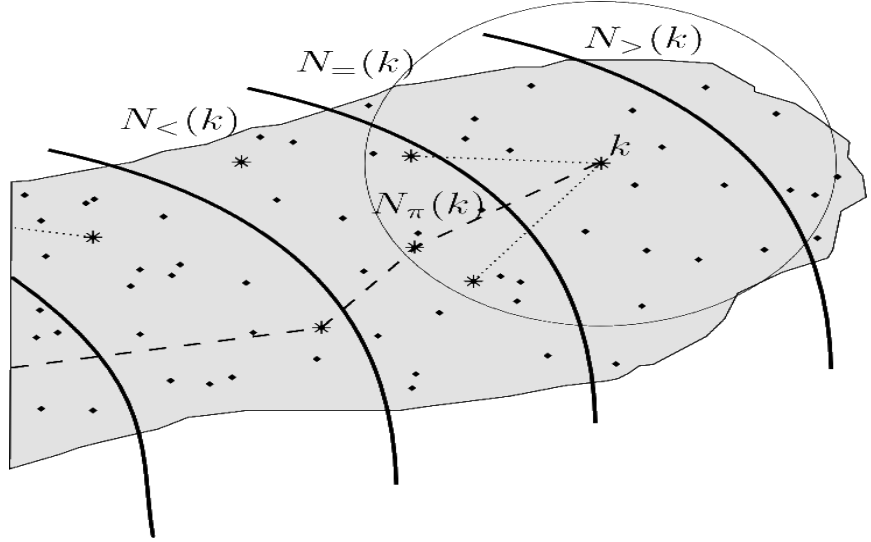

Fig. 3. Building $\mathbf{C h n}(\mathbf{k})$ element within neighborhood.

towards the BS. Neighbors that belong to a $N_{\pi}(k)$ set are selected from $N_{<}(k)$ set, so $N_{\pi}(k)$ consists of some nodes $i \in N_{<}(k)$. That implies (fig.3):

$$
N_{\pi}(k) \subset N_{<}(k)
$$

\section{FINAL REMARKS}

Large bunch of routing and communication algorithms for Wireless Sensor Networks is energy aware or cluster oriented with neighborhood changing very rarely. Our proposal is also based on nodes cooperation within a neighborhood but instead of using cluster heads for data transmission it takes advantage of a conceptual framework of directed cooperation. Such approach comprehends many sophisticated distributed algorithms in a universal and flexible way that is suitable for analyses.

Any decision and action that is undertaken within the WSN is actually performed by nodes based on their information about their vicinity (within a neighborhood). Relation based approach ensures coincidence of local node activity with globally defined requirements as directed cooperation is. In order to meet this global requirement, every single network node gathers all the information that allows aligning the transmission flow with the preferred direction. Hop distance and relations proposed in this paper ensure that the whole communication is directed towards the $\mathrm{BS}$, there are multiple paths to route messages from a particular node to the BS, and some nodes can be excluded from routing if necessary.

\section{REFERENCES}

[1] D. Braginsky and D. Estrin, Rumor Routing Algorithm for Sensor Networks, Proc. of the 1-st Workshop on Sensor Networks and Applications, Atlanta,GA, October 2002.

[2] L. Briesemeister, G. Hommel, Localized Group Membership Services for Ad Hoc Networks, Int. Workshop on Ad Hoc Networking (IWAHN), pp 94-100, AUG 2002.

[3] M. Burmester, T.V. Le, A. Yasinsac, Adaptive gossip protocols: Managing security and redundancy in dense ad hoc networks, Ad Hoc Networks, Volume 5, Issue 3,pp. 313-32, 3April 2007.

[4] Z. Chaczko, P. Moses, C. ChiuM, Cooperative EKMs for Wireless Sensor Networks, 12th Int. Conf. on Computer Aided Systems Theory, February 15-20, 2009, Las Palmas de Gran Canaria, Canary Islands, Spain. 
[5] A.Datta, S.Quarteroni, K.Aberer, Autonomous Gossiping: A SelfOrganizing Epidemic Algorithm for Selective Information Dissemination in Wireless Mobile Ad-Hoc Networks, LNCS, Springer Berlin / Heidelberg, vol.3226/2004, Semantics of a Networked World, pp.126-143, 2004 .

[6] Q.Fang, J.Gao, L.J.Guibas, V. de Silva, L.Zhang, GLIDER: gradient landmark-based distributed routing for sensor networks, INFOCOM 2005, 24th Annual Joint Conference of the IEEE Computer and Communications Societies. 13-17 March 2005, vol.1, pp.339-350, 2005.

[7] J.Jaroń, Systemic Prolegomena to Theoretical Cybernetics, Scient. Papers of Inst. of Techn. Cybernetics, Wrocław Techn. Univ., no. 45, Wrocław, 1978

[8] A.Lim, Distributed Services for Information Dissemination in SelfOrganizing Sensor Networks, Distributed Sensor Networks for Real-Time Systems with Adaptive Reconfiguration, Journal of Franklin Institute, Elsevier Sciences Publisher, vol.338, pp.707-727, 2001.

[9] A. Manjeshwar, D.P. Agrawal, TEEN: A Routing Protocol for Enhanced Efficiency in Wireless Sensor Networks ,Parallel and Distributed Processing Symposium, International, vol. 3, pp. 30189a, 15th International Parallel and Distributed Processing Symposium (IPDPS'01) Workshops, 2001.

[10] J.Nikodem, R.Klempous, Z.Chaczko, Modelling of immune functions in a wireless sensors network. 20th European Modeling and Simulation Symposium. EMSS 2008, Campora S. Giovanni, Italy, 2008

[11] J. Nikodem, Relational Approach Towards Feasibility Performance for Routing Algorithms in Wireless Sensor Network, Proc. of the Conference on Dependability of Computer Systems, DepCoS - RELCOMEX 2009, Poland, 2009.

[12] J. Nikodem, Designing Communication Space in Wireless Sensor Net work Based on Relational Attempt, EUROCAST'2009, 12 International Conference on Computer Aided Systems Theory and Technology, in: Lecture Notes in Computer Science (in printing), Springer-Verlag Berlin Heidelberg 2009.

[13] J.Sung-Min, H.Young-Ju, C.Tai-Myoung, The Concentric Clustering Scheme for Efficient Energy Consumption in the PEGASIS, The 9th International Conference on Advanced Communication Technology, Vol.1, page(s): 260-265, 2007.

[14] Vakil S, Liang B.: Balancing Cooperation and Interference in Wireless Sensor Networks, IEEE Communications Society on Sensor and Ad Hoc Communications and Networks,SECON '06, vol.1, pp.198-206, 2006.

[15] M. Welsh, G. Mainland, Programming Sensor Networks Using Abstract Regions, First USENIX/ACM Symp. on Networked Systems Design and Implementation, (NSDI'04) March 2004. 
Conferences: BROADBANDCOM2009 notification for paper 211

(12 of 1338)

\section{Delete | Reply | Reply to All | Forward | Redirect | Blacklist | Whitelist | Message Source | Save as | Print | Report as Spam Back to Conferences}

Date: Thu, 4 June 2009 18:49:12+0000

From: BROADBANDCOM2009<BroadbandCom2009@easychair.org>

To: Zenon Chaczko<zenon@eng.uts.edu.au>

Subject: BROADBANDCOM2009 notification for paper 211

\section{Dear Zenon,}

Thank you for your submission, "Multihop Communication in WSN Based on Directed Cooperation", to BroadBandCom 2009. I am delighted that we are able to conditionally accept your paper for publication.

All submissions were reviewed by at least three members of the BroadBandCom 2009 Program Committee, and their comments are included below. Please take the below comments by reviewers into account when updating your submission. Because your paper is conditionally accepted, it means that your revised paper will be compared to the reviewer comments, and selected reviewers may be asked to check your paper against their original reviews. If the reviewers are unsatisfied with your revisions, the paper may not be accepted.

Due to the tight timelines for publication, we ask that you upload your camera-ready copy to EasyChair (replacing your submission) by the end of July 15. If your paper is not uploaded by this time, we may be unable to include it in the proceedings, and your paper will not be presented at the conference. Details regarding presentation format and venue are forthcoming.

Congratulations again on your submission.

Best regards,

BROADBANDCOM2009 General Chair

PAPER: 211

REVIEW 1

TITLE: Multihop Communication in WSN Based on Directed Cooperation

OVERALL RATING: 0 (borderline paper)

ADDRESSES CONFERENCE SCOPE: 3 (fair)

TECHNICAL MERIT: 2 (poor)

WRITING AND ORGANIZATION: 4 (good)

This paper discusses an approach for Wireless Sensors to identify its neighbors around them.

This is a hot research topic in last ten years. However, no new idea can be found in the paper.

The proposed approach is not verified. There are many network simulation software available. The authors can use any of them to conduct some experiments. Then, the paper will

be much stronger.

PAPER: 211

TITLE: Multihop Communication in WSN Based on Directed Cooperation

OVERALL RATING: 2 (accept)

ADDRESSES CONFERENCE SCOPE: 3 (fair)

TECHNICAL MERIT: 3 (good) 
WRITING AND ORGANIZATION: 4 (good)

The authors propose a new abstraction to represent cooperation in sensor networks. Their proposal allows for the construction of a multi-hop path to the base station, as opposed to most approaches that rely on a single hop one.

WebMail :: Conferences: BROADBANDCOM2009 notification for paper 211

https://webmail.eng.uts.edu.au/horde/imp/message.php?index $=3300$ 
VERSIT | CENTRAL EUROPEAN SCIENCE PUBLISHERS

Journal Home Account Support

\section{Journal Article}

\section{Welcome!}

To use the personalized features of this site, please log in or register.

If you have forgotten your username or password, we can help.

Institutional Login

\section{Journals Home}

About Us

Contact Us

Browse Publications

Versita Open

Versita Collection

\section{My Menu}

Publication Alerts

Marked Items

Alerts

Order History

\section{Saved Items}

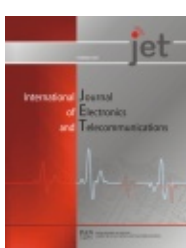

Directed Cooperation in Multihop Wireless Sensors Network

Add to marked items

Journal

Publisher

ISSN

Versita, Warsaw

0867-6747

Issue Volume 56, Number 1 / March 2010

Pages 7-12

DOI

$10.2478 / v 10177-010-0001-8$

Online Date Friday, July 30, 2010

PDF (1.1 MB)

\section{Authors}

Jan Nikodem ${ }^{1}$, Ryszard Klempous ${ }^{1}$, Maciej Nikodem ${ }^{1}$, Marek Woda ${ }^{1}$, Zenon Chaczko ${ }^{2}$

${ }^{1}$ Faculty of Electronics, Wrocław University of Technology, Wyspianskiego 27, 50-370 Wrocław, Poland

2 University of Technology Sydney, PO Box 123, Broadway NSW 2007, Australia

\section{Abstract}

This paper proposes a relational abstraction for Wireless Sensors Network where node can identify its neighbors around it. Because of limited radio link range only some of nodes have a direct contact with the base station and transmission is carried out in a multihop way so information is send from one node to another towards the BS. We propose a

relation $\pi$ that represents cooperation between nodes and takes advantages of topological properties of the network. Based on the hop-distance from the BS any node $k$ can determine a set $N<(k)$ that consists of nodes to which/ $k$ should send messages in order to retain a data-flow direction towards the BS.

\section{Keywords}

Wireless sensors network, directed cooperation, relations

Show References

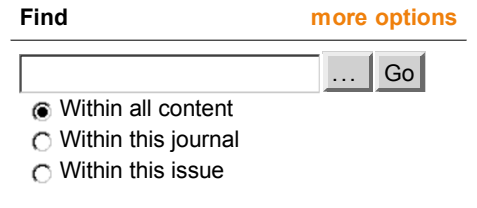

Export this chapter

RIS | Text 